\title{
Subclinical Hypothyroidism and Depression: A Systematic Review and Meta-Analysis
}

\author{
Rong Tang ${ }^{1+}$, Jian Wang ${ }^{2+}$, Lili Yang ${ }^{3}$, Xiaohong Ding ${ }^{4}$, Yufan Zhong ${ }^{5}$, Jiexue Pan ${ }^{6}$, \\ Haiyan Yang ${ }^{6}$, Liangshan $\mathrm{Mu}^{6}$, Xia Chen ${ }^{6 *}$ and Zimiao Chen ${ }^{1 *}$
}

${ }^{1}$ Department of Endocrinology, The First Affiliated Hospital of Wenzhou Medical University, Wenzhou, China, ${ }^{2}$ Department of Hand Surgery and Peripheral Neurosurgery, The First Affiliated Hospital of Wenzhou Medical University, Wenzhou, China, ${ }^{3}$ Department of Radiology, The Second Affiliated Hospital and Yuying Children's Hospital of Wenzhou Medical University, Wenzhou, China, ${ }^{4}$ The First Clinical Medical School, Wenzhou Medical University, Wenzhou, China, ${ }^{5}$ The Second Clinical Medical School, Wenzhou Medical University, Wenzhou, China, ${ }^{6}$ Reproductive Medicine Center, The First Affiliated Hospital of Wenzhou Medical University, Wenzhou, China

OPEN ACCESS

Edited by:

Alessandro Antonelli,

University of Pisa, Italy

Reviewed by:

Trevor Edmund Angell,

University of Southern California,

United States

Anthony Martin Gerdes,

New York Institute of Technology,

United States

Roberto Vita

University of Messina, Italy

*Correspondence:

Zimiao Chen

zimiaochen@163.com

Xia Chen

Chuangchi@163.com

${ }^{\dagger}$ These authors have contributed equally to this work

Specialty section:

This article was submitted to

Thyroid Endocrinology,

a section of the journal

Frontiers in Endocrinology

Received: 21 August 2018 Accepted: 10 May 2019

Published: 04 June 2019

Citation:

Tang R, Wang J, Yang L, Ding $X$, Zhong Y, Pan J, Yang H, Mu L, Chen X and Chen $Z$ (2019) Subclinical

Hypothyroidism and Depression: A Systematic Review and Meta-Analysis.

Front. Endocrinol. 10:340.

doi: 10.3389/fendo.2019.00340
Background: Thyroid function is closely associated with neuropsychological functions, including mental state and cognitive functions. Although thyroid function is routinely examined in persons with depressive symptom, the association between subclinical hypothyroidism $(\mathrm{SCH})$ and depression remains inconclusive.

Objective: This systematic review and meta-analysis aimed to evaluate the risk of depression in persons with $\mathrm{SCH}$.

Methods: The PubMed, Embase, and Web of Science databases were searched up to August 2018. The primary outcome was the prevalence of depression, as evaluated by various types of self-reported depression scales. Odds ratios (ORs) were calculated to compare the risk of depression between persons with $\mathrm{SCH}$ and those with euthyroidism.

Results: Twenty-one studies were included in the systematic review, with a total of 103,375 subjects from 7 studies being pooled for the meta-analysis to evaluate the risk of depression. The meta-analysis showed that persons with $\mathrm{SCH}$ had a significantly elevated risk of depression than persons with euthyroidism $(\mathrm{OR}=1.78,95 \%$ confidence interval $[C l]: 1.11-2.86, P=0.02)$. No publication bias was found, as indicated by Egger's test $(t=-0.49, P=0.647)$ and Begg's test $(z=-0.15, P=0.881)$. In addition, the funnel plot showed a symmetric distribution.

Conclusions: This meta-analysis demonstrated that $\mathrm{SCH}$ was positively associated with the risk of depression, especially in persons above 50 years of age, suggesting it is necessary to pay close attention to depressive symptoms in persons with $\mathrm{SCH}$.

Keywords: subclinical hypothyroidism, depression, thyroid, neuroendocrinology, thyroxine

\section{INTRODUCTION}

Thyroid function is closely associated with neuropsychological functions, including mental state and cognitive functions (1). Subclinical hypothyroidism ( $\mathrm{SCH}$ ) is defined as a condition with elevated thyroid-stimulating hormone (TSH) and normal free thyroxine (T4) levels (2). The prevalence of SCH is approximately $4-10 \%$ in adults (3) and is associated with neuropsychological dysfunction (4). 
In recent years, an increased number of studies investigated the association between SCH and depression $(5,6)$. However, the findings of these reports could not reach a consensus. Some studies reported that the prevalence of depression is higher in persons with $\mathrm{SCH}$ than in euthyroid people, $(4,7)$ while others found the risk for depression is comparable between the two population $(6,8)$. Therefore, this systematic review aimed to summarize the existing information and evaluate the risk of depression in persons with $\mathrm{SCH}$.

\section{METHODS}

\section{Study Design}

This meta-analysis was performed according to the Preferred Reporting Items for Systematic Reviews and Meta-Analysis (PRISMA) (9) and the Meta-analysis of Observational Studies in Epidemiology (MOOSE) (10).

\section{Participants}

$\mathrm{SCH}$ participants were diagnosed according to the specific diagnostic criteria in each included study (Table 1) and the controls for these studies included participants with euthyroid function. In addition, all participants were not limited by age group, geographical region, or race.

\section{Search Strategy}

The PubMed, Embase, and Web of Science literature databases were searched, without restriction of language, for publications up to August 2018. In order not to miss potential studies, we use "hypothyroidism" as a keyword instead of "subclinical hypothyroidism" to expand retrieval results because some articles may use similar terms like "mild hypothyroidism" or "slight hypothyroidism." This searched keyword was combined with "depression" and "depressive symptom" in the "title and abstract" column. The complete search items were "[(hypothyroidism) AND (depression OR depressive symptom)]" in PubMed and Web of Science, and hypothyroidism AND (depression OR "depressive symptom") in Embase.

\section{Studies Sections and Data Extraction}

Selected studies were eligible if they met the following criteria: (1) SCH was confirmed by elevated TSH levels and circulating thyroid hormones within the normal range; and (2) the study was a cross-sectional, case-control, or cohort study. Studies were excluded if: (1) the title and/or abstract were not appropriate for the objective of this review; (2) the use of antidepressant drugs was not allowed in a specific period before each trial; and (3) the article was a review, case report, non-human study, or an abstract or conference proceeding, or if it contained unpublished data. Two reviewers screened potential articles independently and a third reviewer made the final decision when discrepancies were found with a specific study.

Data, including study name, characteristics of the study population (age, race, and sex), main conclusion(s), diagnostic criteria, number of $\mathrm{SCH}$ participants with depression and the total number of $\mathrm{SCH}$ patients, the number of euthyroid participants with depression, the total number of euthyroid participants were extracted. If published papers only provided the

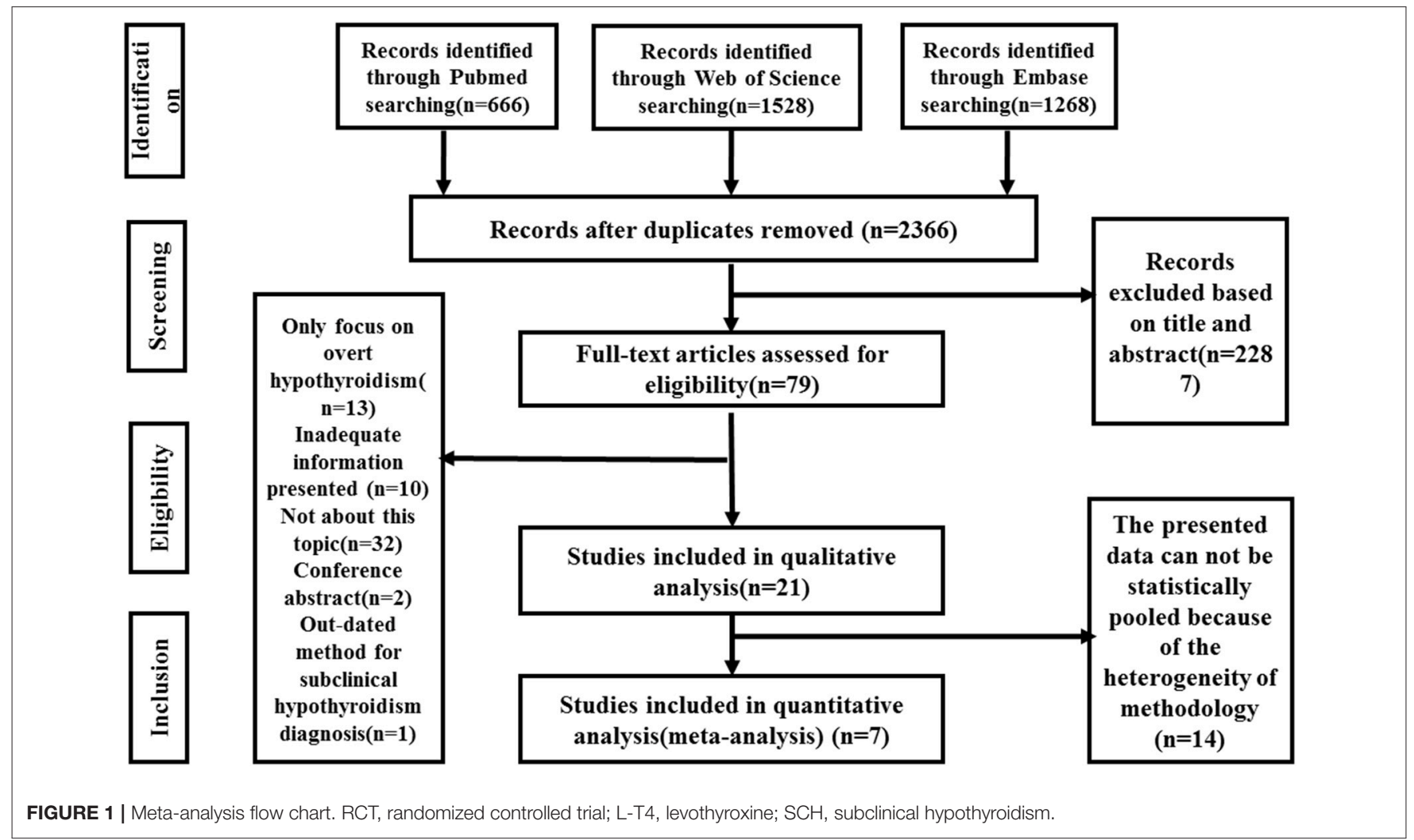


TABLE 1 | Characteristics of included studies.

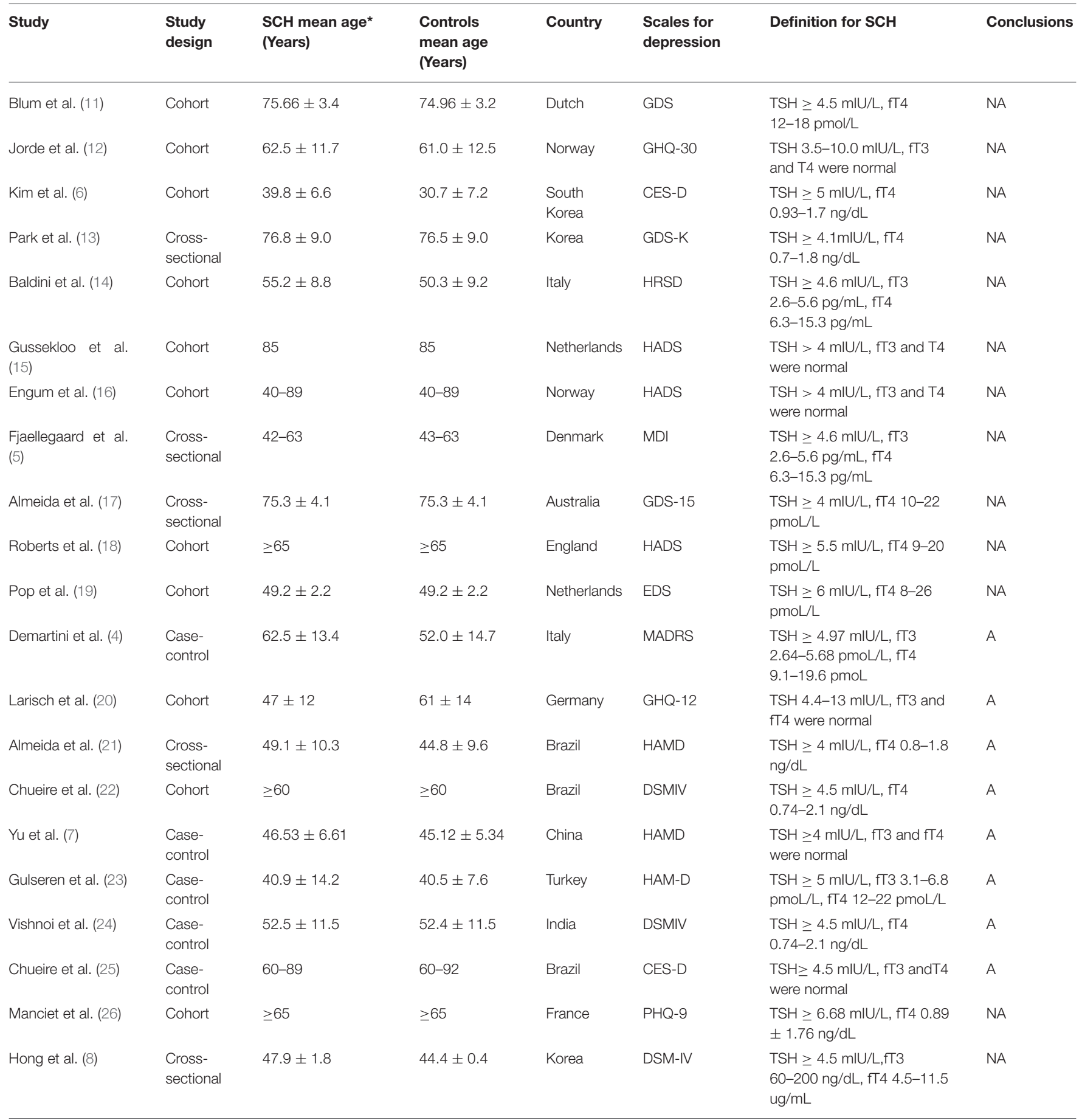

"The mean age of the enrolled patients.

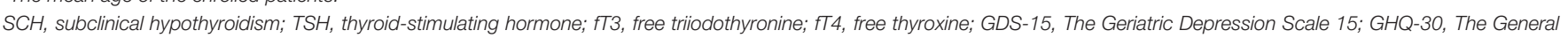

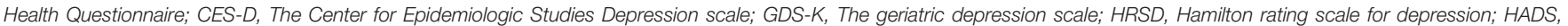

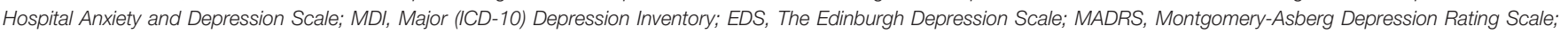

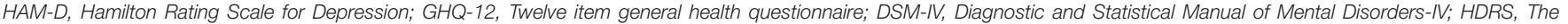
Hamilton Depression Rating Scale; RDC, The Research Diagnostic Criteria; NA, No association; A, Significant association. 


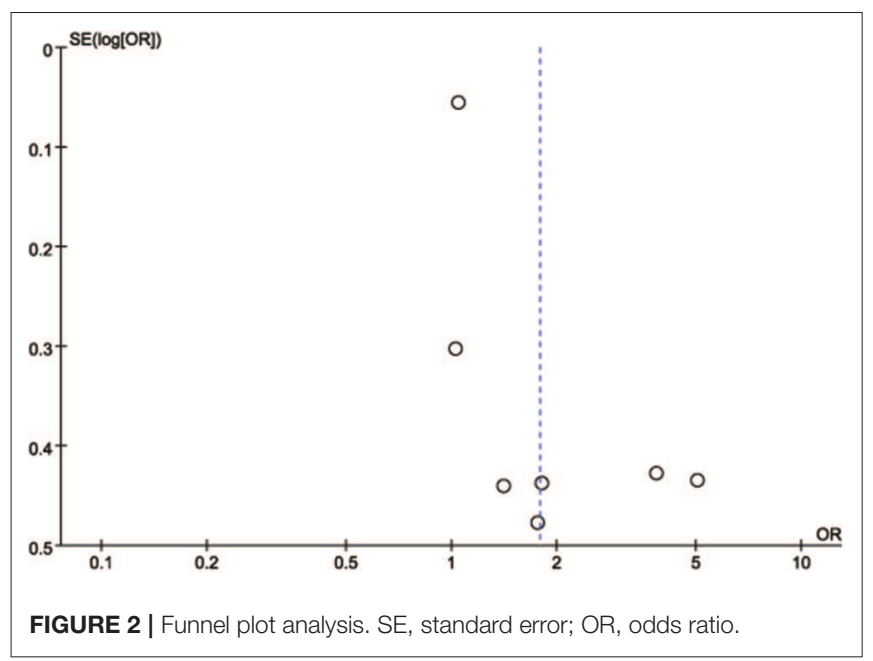

percentage of depressive symptoms, the evaluator calculated the numbers of participants by the calculator in Revman version 5.3 (The Cochrane Collaboration, Copenhagen, Denmark).

\section{Statistical Analysis}

The literature quality evaluation was conducted by means of the Newcastle-Ottawa Quality Assessment Scale (27). Two reviewers conducted this step independently and disagreements were resolved by consensus. The presence of publication bias was assessed by the combination of Egger's test, Begg's test, and visual inspection of the funnel plot.

Odds ratio (OR) and 95\% confidence intervals (CIs) were calculated for the outcome of depression, in which the prevalence of depressive symptoms in $\mathrm{SCH}$ participants and a normal population was assessed. The reason for the choice of OR was due to the retrospective design of this meta-analysis based on published studies that varied in design, subject populations, primary outcome measures, and research quality (28).

A forest plot was used to present the pooled OR value, and heterogeneity was evaluated using the $I^{2}$ statistic. Due to the potential clinical heterogeneity, a random-effect model was chosen to synthesize data instead of a fixed-effect model. Heterogeneity was considered significant if the $I^{2}$ value was over $25 \%$. All reported test results were two-tailed and a $P$ value $<0.05$ was considered statistically significant. All data analyses were performed with Revman 5.3.

\section{RESULTS}

\section{Study Selection}

A flow chart of the meta-analysis is shown in Figure 1. Initially, a total of 3,462 studies were identified by the search strategy. After duplicates removal, 2,366 studies received screening based on the title or abstract. Next, 79 studies were assessed for eligibility by reviewing the full-text article. In addition, 58 studies were excluded for the following reasons: only focusing on overt hypothyroidism $(n=13)$; inadequate information presented $(n=$ $10)$; not related to our study topic $(n=32)$; conference abstract $(n=2)$; outdated method for SCH diagnosis $(n=1)$. A total of 21 studies were included in the qualitative analysis. Among these studies, 14 studies presented the mean scores of depression scales of each group or only provided the regression results without details, which hinders access to data analysis. Finally, seven studies were eligible and included in the final quantitative analysis (meta-analysis).

\section{Characteristics of Included Studies}

The characteristics of 21 studies were presented in Table 1, which included 11 cohort, 5 cross-sectional, and 5 case-control studies with mean participant age of 39.8-92 years. Participants in these studies came from different regions, including America, Europe, and Asia. Studies from Korea, the Netherlands, and Norway showed null associations between SCH and depression, while all the studies from Brazil showed significant associations. Heterogeneity among the studies was caused by different SCH diagnosis criteria and variations in the use of depression scales for assessment. Serum TSH cut-off values for the diagnosis of SCH range from 3.5 to $6 \mathrm{mIU} / \mathrm{L}$. Various scales were used for detecting depressive symptoms, including the geriatric depression scale (GDS) (29), the hospital anxiety and depression scale (HADS) (30), and the Hamilton depression rating scale (HAMD) (31). Among these 21 studies, 8 showed significant associations between SCH and depression, while the other 13 did not show this relationship.

\section{Risk of Bias}

The quality of the literature for the analyzed studies was evaluated using the Newcastle-Ottawa Quality Assessment Scale. Overall, the quality for each study was found to be "good" as indicated by 6-plus scores. The main drawback of these studies was that participants in some cohorts were not representative and outcome assessment was self-reported (questionnaires); however, this is typically inevitable for these types of studies. For crosssectional studies, the common deficiency was the lack of a direct description of response rates and the completeness of data collection that could be determined. The case-control study by $\mathrm{Yu}$ et al. (7) also lacked the typical case representation due to the enrolled participants having been diagnosed with Graves' disease before the trial, but the overall score was still intermediate (6 scores).

No publication bias was found from Egger's test $(t=-0.49$, $P=0.647)$ or Begg's test $(z=-0.15, P=0.881)$, and the funnel plot showed a symmetric distribution (Figure 2). Obvious heterogeneity $\left(I^{2}=75 \%\right)$ was detected and not diminished after a sensitivity analysis by removing a single study at a time in an iterated manner.

\section{The Risk of Depression in Persons With $\mathrm{SCH}$}

Overall, a significant difference was found for the synthesized endpoint (OR $=1.78,95 \% \mathrm{CI}: 1.11-2.86, P=0.02)$, and the prevalence of depression in SCH participants $(8.6 \%, 463 / 5,382)$ was slightly higher than that of the control group $(7.5 \%$, $7,311 / 97,993$ ) (Figure 3). Considering the influence of age on the results, a subgroup analysis including 4 studies, whose participants were characterized with a mean age of $<50$ years, was performed. No significant difference was observed for the risk of depression between $\mathrm{SCH}$ participants and the control group 


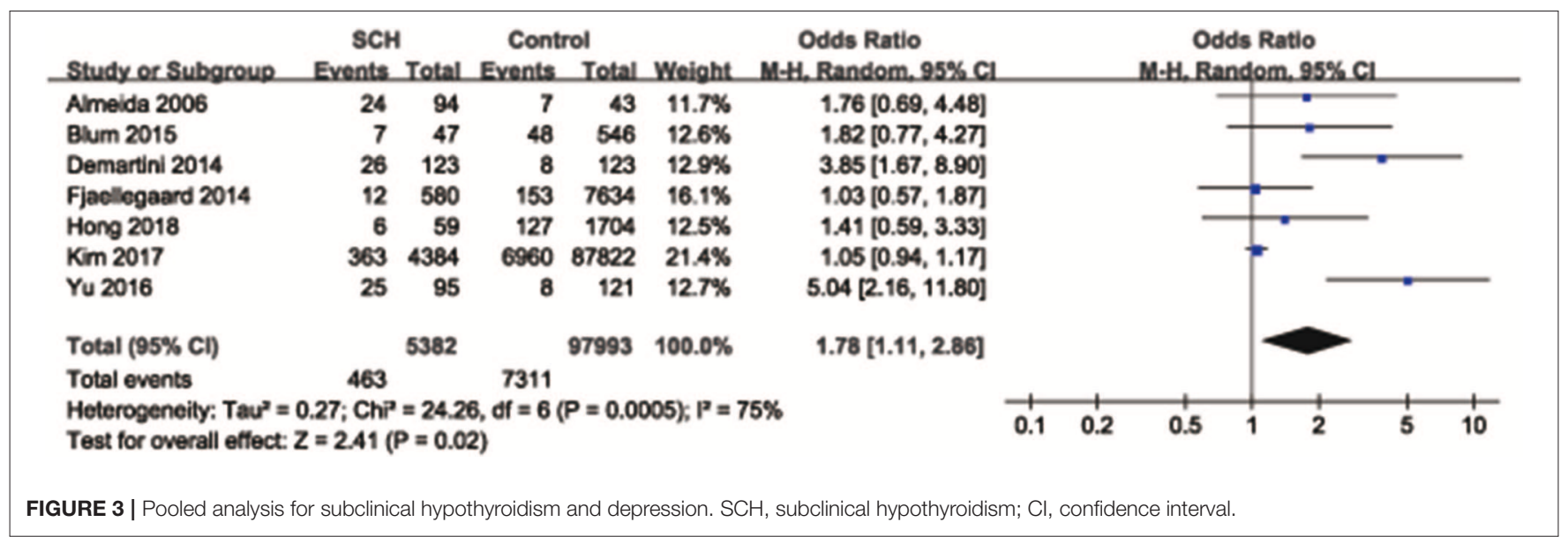

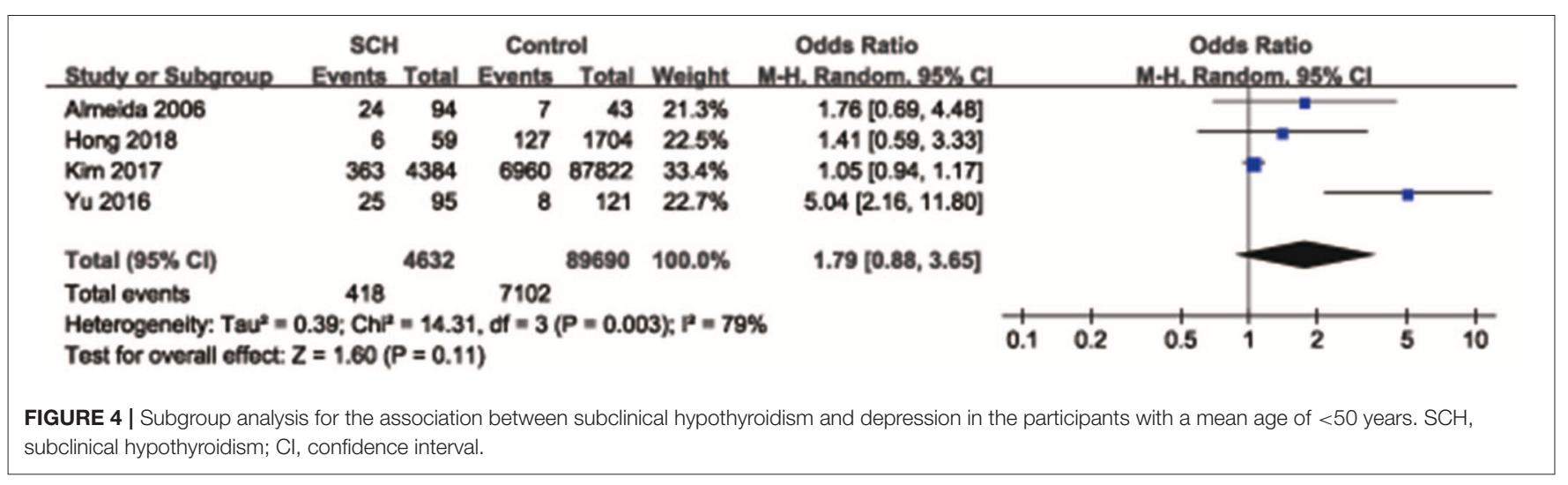

in participants with a mean age $<50$ years $(\mathrm{OR}=1.79,95 \% \mathrm{CI}$ : $0.88-3.65, P=0.11$ ) (Figure 4).

\section{DISCUSSION}

\section{Summary of Main Findings}

This systematic review and meta-analysis demonstrated that the prevalence of depression is slightly higher in persons with $\mathrm{SCH}$, especially in persons above 50 years of age, suggesting subclinical hypothyroidism might be a risk factor for depression.

Previously, many studies investigated the relationship between $\mathrm{SCH}$ and depression; however, these studies showed conflicting conclusions. Recently, a large cohort study conducted by Kim et al. analyzed a total of 92,206 young to middle-aged adults and found no link between SCH and depression (6). In the above study, TSH, free T4, and free T3 were measured, and the Epidemiologic Studies-Depression Scale (CES-D) was used for assessments at least twice a year. However, the limitation of aforementioned study was that the participants primarily were middle-aged and generally healthy men and women from Korea, which may not be generalizable to other ethnic or age groups. An interesting finding in our review was that all studies from Korea $(6,13,32)$, the Netherlands $(15,19)$, and Norway $(12,16)$ showed no association between SCH and depression, while studies from Brazil $(22,25,33)$ did show significant associations, which suggests that regional differences, including economy, lifestyle, and diet, play key roles in these observational studies.

Another interesting phenomenon was observed in terms of age stratification. In our qualitative analysis, we found that $22 \%(2 / 9)$ of the included studies, which enrolled participants aged over 60 years, showed a positive correlation between depression and $\mathrm{SCH}(4,22,25)$; while $50 \%$ (4/8) of the studies, which had participants aged $<60$ years, reported that the presence of this association $(7,23,24,33)$. However, these qualitative observations are limited by different sample sizes. In order to further investigate whether age influences the risk of depression in SCH participants quantitatively, a subgroup analysis including 4 studies in which the mean participant age was $<50$ years was conducted, showing no association between depression and $\mathrm{SCH}$. This result indicates that only older participants are more susceptible to $\mathrm{SCH}$. Thus, in elderly populations, depression is more likely to occur in participants with SCH. This phenomenon may depend on the higher reactivity of 5-HT system to the decreased T4 level in elder people compared with young individuals (34).

Fourteen studies were excluded in our final meta-analysis because they utilized mean depressive scores or other methodologies to present their results in the final publication, making it impossible to meta-analyze these data. Among these 
studies, Park et al. (13) enrolled 944 Korean people aged at least 65 years old, who did not have any thyroid diseases in their study, with neuropsychological function being evaluated by GDS-K score and serum TSH and free thyroxine also being measured. In their study, mean scores of depressive symptoms were comparable between participants with SCH and euthyroid participants. However, a recent study (24) reported the opposite conclusion. In this study, HAM-D scores were significantly higher for participants with $\mathrm{SCH}$ as compared to controls. In addition, after 2 months of levothyroxine (L-T4) treatment, they observed a significant fall in TSH levels and HAM-D scores. Several studies showed similar results, illustrating that a "worse" score is more likely in persons with $\mathrm{SCH}$. Therefore, the use of various scales for the detection and quantification of depression may be limited by methodological heterogeneity, disturbing the horizontal comparisons among different studies. We here recommend the CES-D scale for future studies involved in this area (35), which is generally recommended for the primary screening of depression in community care or primary care, as it has shown great sensitivity and specificity in both young and elderly populations (36).

Although some studies reported that L-T4 treatment has the benefit of improving depressive symptoms, the pathophysiological role of thyroid hormones in depression remains unclear (37-40). A biologically plausible theory is that L-T4 may exert modulatory effects on the illness via an increase in serotonergic neurotransmission, specifically by reducing the sensitivity of 5-HT1A (5-hydroxytryptamine 1A) autoreceptors in the raphe area and by increasing 5-HT2 receptor sensitivity (41). High serum TSH levels are associated with depression in the elderly, which suggests that increased TSH could disturb the stability of 5-HT system and thereby facilitates the development of depression in SCH participants (22). This effect is partially explained by the efficacy of L-T4 treatment observed in some studies.

This meta-analysis demonstrates that there is a higher prevalence of depression in persons with $\mathrm{SCH}$, raising some suggestions for clinical practice. First, timely screening and intervention of depressive symptoms are of great importance in persons with $\mathrm{SCH}$. Second, our findings strongly support the routine examination of thyroid function in participants with depression in the clinic. Third, L-T4, which is the suggested treatment for hypothyroidism, may be effective in improving depressive symptoms in participants with $\mathrm{SCH}$ if the association between the presence of SCH and depression will be proved. Therefore, well-conducted and large-scale random controlled trials are expected to verify the therapeutic effect on depressive symptoms by L-T4 (12, 37-40, 42-44).

\section{REFERENCES}

1. Feldman AZ, Shrestha RT, Hennessey JV. Neuropsychiatric manifestations of thyroid disease. Endocrinol Metab Clin North Am. (2013) 42:453-76. doi: 10.1016/j.ecl.2013.05.005

2. Baumgartner C, Blum MR, Rodondi N. Subclinical hypothyroidism: summary of evidence in 2014. Swiss Med Wkly. (2014) 144:w14058. doi: $10.4414 /$ smw.2014.14058

\section{Strengths and Limitation}

To the best of our knowledge, this is the first systematic review and meta-analysis aimed to verify the association between subclinical hypothyroidism and depression. The total number of the cases and controls was sufficiently large to support our conclusions. However, several limitations should be taken into consideration. First, the age of subjects in the included studies was not restricted to a specific range. Second, the included reports were designed as observational studies (case control, cross-sectional, and cohort studies) with different data collection methods (retrospective and prospective). Third, there may be possible bias due to the heterogeneity of the analyzed studies in terms of the definition of $\mathrm{SCH}$ and the evaluation of depression. Nonetheless, it is recommended that each laboratory should establish their own cut-off value to evaluate $\mathrm{SCH}$ based on the age, region, ethnic factors. Thus, the heterogeneity pertaining to the different cut-off value of TSH for the diagnosis of $\mathrm{SCH}$ is acceptable. In addition, $\mathrm{SCH}$ may result from antithyroid treatment in patients with Graves' disease. In such patients, neurological disturbances may stem from either the hyperthyroid state or the iatrogenic hypothyroidism that ensues from antithyroid treatment (45). Future studies should focus on the underlying mechanism behind the association between SCH and depression and whether depression or other neuropsychological conditions could be treated through the supplement of L-T4.

\section{Conclusion}

In conclusion, our study demonstrated that depression is positively associated with $\mathrm{SCH}$, suggesting that it is necessary to for clinicians to pay special attention to depression symptoms in patients with SCH. Future studies should focus on whether depression or other neuropsychological conditions could be improved by treating thyroid dysfunction and any potential mechanism.

\section{AUTHOR CONTRIBUTIONS}

RT, JW, and LY: data analysis. XD and YZ: data extraction. JP, HY, and LM article screening. XC and ZC: paper writing and review.

\section{FUNDING}

This study was supported by Wenzhou Municipal Science and Technology Bureau (Y20170122 and Y20160037), Zhejiang Traditional Chinese Medicine Foundation (2017ZQ020), and Zhejiang Provincial Medical and Health Research Project (2017KY462).

3. Biondi B, Cooper DS. The clinical significance of subclinical thyroid dysfunction. Endocr Rev. (2008) 29:76-131. doi: 10.1210/er.2006-0043

4. Demartini B, Ranieri R, Masu A, Selle V, Scarone S, Gambini O. Depressive symptoms and major depressive disorder in patients affected by subclinical hypothyroidism a cross-sectional study. J Nerv Mental Dis. (2014) 202:603-7. doi: 10.1097/NMD.0000000000000168

5. Fjaellegaard K, Kvetny J, Allerup PN, Bech P, Ellervik C. Well-being and depression in individuals with subclinical hypothyroidism and thyroid 
autoimmunity-a general population study. Nord J Psychiatry. (2015) 69:73-8. doi: 10.3109/08039488.2014.929741

6. Kim JS, Zhang Y, Chang Y, Ryu S, Guallar E, Shin Y-C, et al. Subclinical hypothyroidism and incident depression in young and middleage adults. J Clin Endocrinol Metab. (2018) 103:1827-33. doi: 10.1210/ jc.2017-01247

7. Yu J, Tian AJ, Yuan X, Cheng XX. Subclinical hypothyroidism after 131Itreatment of Graves' disease: a risk factor for depression? PLoS ONE. (2016) 11:e0154846. doi: 10.1371/journal.pone.0154846

8. Hong JW, Noh JH, Kim D-J. Association between subclinical thyroid dysfunction and depressive symptoms in the Korean adult population: The 2014 Korea National Health and Nutrition Examination Survey. PLoS ONE. (2018) 13:e0202258. doi: 10.1371/ journal.pone.0202258

9. Shamseer L, Moher D, Clarke M, Ghersi D, Liberati A, Petticrew M, et al. Preferred reporting items for systematic review and meta-analysis protocols (PRISMA-P) 2015: elaboration and explanation. BMJ. (2015) 350:g7647. doi: 10.1136/bmj.g7647

10. Stroup DF, Berlin JA, Morton SC, Olkin I, Williamson GD, Rennie $\mathrm{D}$, et al. Meta-analysis of observational studies in epidemiology-a proposal for reporting. JAMA. (2000) 283:2008-12. doi: 10.1001/jama.28 3.15.2008

11. Blum MR, Wijsman LW, Virgini VS, Bauer DC, den Elzen WP, Jukema JW, et al. Subclinical thyroid dysfunction and depressive symptoms among the elderly: A prospective cohort study. Neuroendocrinology. (2016) 103:291-9. doi: $10.1159 / 000437387$

12. Jorde R, Waterloo K, Storhaug H, Nyrnes A, Sundsfjord J, Jenssen TG. Neuropsychological function and symptoms in subjects with subclinical hypothyroidism and the effect of thyroxine treatment. J Clin Endocrinol Metab. (2006) 91:145-53. doi: 10.1210/jc.2005-1775

13. Park YJ, Lee EJ, Lee YJ, Choi SH, Park JH, Lee SB, et al. Subclinical hypothyroidism $(\mathrm{SCH})$ is not associated with metabolic derangement, cognitive impairment, depression or poor quality of life (QoL) in elderly subjects. Arch Gerontol Geriatr. (2010) 50:e68-73. doi: 10.1016/j.archger.2009.05.015

14. Baldini IM, Vita A, Mauri MC, Amodei V, Carrisi M, Bravin S, et al. Psychopathological and cognitive features in subclinical hypothyroidism. Prog Neuropsychopharmacol Biol Psychiatry. (1997) 21:925-35. doi: 10.1016/S0278-5846(97)00089-4

15. Gussekloo J, van Exel E, de Craen AJ, Meinders AE, Frolich M, Westendorp RG. Thyroid status, disability and cognitive function, and survival in old age. JAMA. (2004) 292:2591-9. doi: 10.1001/ jama.292.21.2591

16. Engum A, Bjoro T, Mykletun A, Dahl AA. An association between depression, anxiety and thyroid function-a clinical fact or an artefact? Acta Psychiatr Scand. (2002) 106:27-34. doi: 10.1034/ j.1600-0447.2002.01250.x

17. Almeida OP, Alfonso H, Flicker L, Hankey G, Chubb SA, Yeap BB. Thyroid hormones and depression: the Health in Men study. Am J Geriatr Psychiatry. (2011) 19:763-70. doi: 10.1097/JGP.0b013e31820dcad5

18. Roberts LM, Pattison H, Roalfe A, Franklyn J, Wilson S, Hobbs R, et al. Is subclinical thyroid dysfunction in the elderly associated with depression or cognitive dysfunction? Ann Intern Med. (2006) 145:573-81. doi: 10.7326/0003-4819-145-8-200610170-00006

19. Pop VJ, Maartens LH, Leusink G, van Son MJ, Knottnerus AA, Ward AM. Are autoimmune thyroid dysfunction and depression related? J Clin Endocrinol Metab.. (1998) 83:3194-7. doi: 10.1210/jc.83.9.3194

20. Larisch R, Kley K, Nikolaus S, Sitte W, Franz M, Hautzel H, et al. Depression and anxiety in different thyroid function states. Horm Metab Res. (2004) 36:650-3. doi: 10.1055/s-2004-825925

21. Almeida C, Brasil MA, Costa AJ, Reis FA, Reuters V, Teixeira P, et al. Subclinical hypothyroidism: psychiatric disorders and symptoms. Rev Bras Psiquiatr. (2007) 29:157-9.

22. Chueire VB, Brito Silva ET, Perotta E, Romaldini JH, Ward LS. High serum TSH levels are associated with depression in the elderly. Arch Gerontol Geriatr. (2003) 36:281-8. doi: 10.1016/S0167-4943(02)00173-5

23. Gulseren S, Gulseren L, Hekimsoy Z, Cetinay P, Ozen C, Tokatlioglu B. Depression, anxiety, health-related quality of life, and disability in patients with overt and subclinical thyroid dysfunction. Arch Med Res. (2006) 37:1339. doi: 10.1016/j.arcmed.2005.05.008

24. Vishnoi G, Chakraborty B, Garda H, Gowda SH, Goswami B. Low mood and response to Levothyroxine treatment in Indian patients with subclinical hypothyroidism. Asian J Psychiatr. (2014) 8:89-93. doi: 10.1016/j.ajp.2013.12.004

25. Chueire VB, Romaldini JH, Ward LS. Subclinical hypothyroidism increases the risk for depression in the elderly. Arch Gerontol Geriatr. (2007) 44:21-8. doi: 10.1016/j.archger.2006.02.001

26. Manciet G, Dartigues JF, Decamps A, Barberger-Gateau P, Letenneur L, Latapie MJ, et al. The PAQUID survey and correlates of subclinical hypothyroidism in elderly community residents in the southwest of France. Age Ageing. (1995) 24:235-241.

27. Wells GA, Shea B, Higgins JP, Sterne J, Tugwell P, Reeves BC. Checklists of methodological issues for review authors to consider when including nonrandomized studies in systematic reviews. Res Synth Methods. (2013) 4:63-77. doi: $10.1002 /$ jrsm. 1077

28. Chang BH, Hoaglin, DC. Meta-Analysis of odds ratios: current good practices. Med Care. (2017) 55:328-35. doi: 10.1097/ mlr.0000000000000696

29. Guerin JM, Copersino ML, Schretlen DJ. Clinical utility of the 15-item geriatric depression scale (GDS-15) for use with young and middle-aged adults. J Affect Disord. (2018) 241:59-70. doi: 10.1016/j.jad.2018.07.038

30. Stern AF. The hospital anxiety and depression scale. Occup Med Oxford. (2014) 64:393-4. doi: 10.1093/occmed/kqu024

31. Wagner S, Helmreich I, Lieb K, Tadic A. Standardized rater training for the Hamilton depression rating scale $[\operatorname{HAMD}(17)]$ and the inventory of depressive symptoms (IDSC30). Psychopathology. (2011) 44:68-70. doi: $10.1159 / 000318162$

32. Kim J-M. Thyroid stimulating hormone, cognitive impairment and depression in an older Korean population. Psychiatry Investig. (2010) 7:264-9. doi: 10.4306/pi.2010.7.4.264

33. Almeida C, Brasil MA, Costa AJ, Reis FA, Reuters V, Teixeira $\mathrm{P}$, et al. Subclinical hypothyroidism: psychiatric disorders and symptoms. Rev Bras Psiquiatr. (2007) 29:157-9. doi: 10.1590/ S1516-44462007000200013

34. Zhou Y, Wang X, Zhao Y, Liu A, Zhao T, Zhang Y, et al. Elevated thyroid peroxidase antibody increases risk of post-partum depression by decreasing prefrontal cortex BDNF and 5-HT levels in mice. Front Cell Neurosci. (2016) 10:307. doi: $10.3389 /$ fncel.2016.00307

35. Andriushchenko AV, Drobizhev M, Dobrovol'skii AV. [A comparative validation of the scale CES-D, BDI, and HADS(d) in diagnosis of depressive disorders in general practice]. Zh Nevrol Psikhiatr Im S S Korsakova. (2003) 103:11-18.

36. Cho MJ, Nam JJ, Suh GH. Prevalence of symptoms of depression in a nationwide sample of Korean adults. Psychiatry Res. (1998) 81:341-52. doi: 10.1016/S0165-1781(98)00122-X

37. Kong WM, Sheikh MH, Lumb PJ, Naoumova RP, Freedman DB, Crook $\mathrm{M}$, et al. A 6-month randomized trial of thyroxine treatment in women with mild subclinical hypothyroidism. Am J Med. (2002) 112:348-54. doi: 10.1016/S0002-9343(02)01022-7

38. Davis JD, Tremont G. Neuropsychiatric aspects of hypothyroidism and treatment reversibility. Minerva Endocrinol. (2007) 32:49-65.

39. Reuters VS, Almeida Cde P, Teixeira Pde F, Vigario Pdos S, Ferreira MM, Castro CL, et al. Effects of subclinical hypothyroidism treatment on psychiatric symptoms, muscular complaints, and quality of life. Arq Bras Endocrinol Metabol. (2012) 56:128-36. doi: 10.1590/S0004-27302012000200006

40. Najafi L, Malek M, Hadian A, Ebrahim Valojerdi A, Khamseh ME, Aghili R. Depressive symptoms in patients with subclinical hypothyroidism-the effect of treatment with levothyroxine: a double-blind randomized clinical trial. Endocr Res. (2015) 40:121-6. doi: 10.3109/07435800.2014.896924

41. Bauer M, Heinz A, Whybrow PC. Thyroid hormones, serotonin and mood: of synergy and significance in the adult brain. Mol Psychiatry. (2002) 7:140-56. doi: 10.1038/sj.mp.4000963

42. Miller KJ, Parsons TD, Whybrow PC, van Herle K, Rasgon N, van Herle A, et al. Memory improvement with treatment of hypothyroidism. Int J Neurosci. (2006) 116:895-906. doi: 10.1080/00207450600550154 
43. Samuels MH, Schuff KG, Carlson NE, Carello P, Janowsky JS. Health status, psychological symptoms, mood, and cognition in L-thyroxine-treated hypothyroid subjects. Thyroid. (2007) 17:249-58. doi: 10.1089/thy.2006.0252

44. Parle J, Roberts L, Wilson S, Pattison H, Roalfe A, Haque MS, et al. A randomized controlled trial of the effect of thyroxine replacement on cognitive function in community-living elderly subjects with subclinical hypothyroidism: the Birmingham Elderly Thyroid study. J Clin Endocrinol Metab. (2010) 95:3623-32. doi: 10.1210/jc.2009-2571

45. Vita R, Mazzi V, Antonelli A, Benvenga S. Antithyroid medications and psychosis. Expert Opin Drug Saf. (2013) 12:865-72. doi: $10.1517 / 14740338.2013 .823397$
Conflict of Interest Statement: The authors declare that the research was conducted in the absence of any commercial or financial relationships that could be construed as a potential conflict of interest.

Copyright $\odot 2019$ Tang, Wang, Yang, Ding, Zhong, Pan, Yang, Mu, Chen and Chen. This is an open-access article distributed under the terms of the Creative Commons Attribution License (CC BY). The use, distribution or reproduction in other forums is permitted, provided the original author(s) and the copyright owner(s) are credited and that the original publication in this journal is cited, in accordance with accepted academic practice. No use, distribution or reproduction is permitted which does not comply with these terms. 